\title{
Densidade básica e dimensões celulares da madeira em clones de Eucalyptus urophylla, aos oito anos de idade
}

\author{
Patrícia da Silva Paulino ${ }^{1}$, Israel Luiz de Lima ${ }^{2 *}$ \\ ${ }^{1}$ Programa de Pós-Graduação em Ciência do Solo, Universidade do Estado de Santa Catarina (UDESC), Lages, SC Brasil. \\ ${ }^{2}$ Instituto Florestal São Paulo, Seção Madeiras e Produtos Florestais, São Paulo, SP, Brasil.
}

\begin{abstract}
RESUMO O uso industrial da madeira do gênero Eucalyptus é altamente relevante no Brasil. Entretanto, são necessárias novas pesquisas de melhoria de produtividade das florestas e qualidade da madeira são indispensáveis. Assim, é necessário testar novos materiais genéticos das diferentes espécies desse gênero. Este estudo teve como objetivo caracterizar a densidade básica e as dimensões celulares da madeira de quatro clones de Eucalyptus urophylla proveniente de plantio, da região de Esplanada, no estado da Bahia (Brasil). Quatro árvores de cada clone, com 8 anos de idade, foram coletadas e dessas foram tomadas duas toras. As mesmas foram desdobradas, retiradas tábuas e reduzidas na forma de amostras para a realização dos ensaios da densidade básica e dimensões celulares. Os resultados mostraram que entre os clones ocorreram diferenças significativas para: densidade básica, frequência de vaso, porcentagem total de vaso, altura de raio e diâmetro de raio. A posição da tora na altura da árvore diferiu somente para a frequência de vaso.
\end{abstract}

Palavras-chave: qualidade da madeira; anatomia da madeira; densidade.

\section{Basic density and cellular dimensions of wood from 8-year old $E$ u- calyptus urophylla clones}

\begin{abstract}
The industrial use of wood from the genus Eucalyptus is highly relevant in Brazil. However, it is necessary further research to improving forest productivity and wood quality. Thus, it is necessary to test new genetic materials from the different species of the genus. This study aimed to characterize the basic density and cellular dimensions the wood Eucalyptus urophylla clones from a plantation in the region of Esplanada, state of Bahia (Brazil). Four trees from each clone were collected and two logs were taken. The logs were sawn, processed in lumbers and reduced in the final shape of samples for tests of basic density and cellular dimensions. The results demonstrated significant differences among the clones for: basic density, vessel frequency, total vessel percentage, radius height and radius diameter. At the height of the tree, the position of the log differed only for the vessel frequency.
\end{abstract}

Keywords: wood quality; wood anatomy; density.

\section{Introdução}

O gênero Eucalyptus é originário da Austrália, e acreditase que as primeiras mudas de espécies de eucaliptos plantadas, no Brasil, foram nos estados do Rio Grande do Sul e Rio de Janeiro, em 1868 (LIMA, 1993). Algumas dessas espécies se adaptaram bem ao clima e aos diferentes tipos de solo do Brasil, sendo versáteis e com larga aplicação no setor industrial. Inicialmente, o melhoramento genético esteve ligado às empresas do setor de celulose, papel, chapas e siderurgia e, posteriormente, começou a ser utilizado no ramo de postes e madeira para estruturas, na construção civil. (LIMA et. al, 2006; TRUGILHO et al. 2006)

Em nível quantitativo, o estudo anatômico das espécies florestais é fundamental, devido à variação significativa que ocorre nas dimensões de seus elementos anatômicos, entre espécie e dentro da mesma espécie. Muitas das espécies do gênero Eucalyptus apresentam madeira com estrutura anatô- 
mica muito homogênea em nível morfológico, o que torna difícil a separação, com base apenas na composição e arranjo estrutural dos constituintes de seu lenho (OLIVEIRA et al., 1999).

Algumas dessas espécies de Eucalyptus possuem madeira de alta densidade básica e bom rendimento na produção de pasta celulósica, tendo uma crescente demanda de suas fibras devido às suas propriedades, que possibilitam a fabricação de produtos com elevada absorção, maciez, opacidade e boa qualidade de impressão (MORA; GARCIA, 2000).

A densidade básica da madeira varia com a idade, altura, direção radial, tem boas relações com diversas propriedades e caraterísticas anatômicas das árvores (GITHIOMI; KARIUKI, 2010). A caracterização tecnológica da madeira de eucalipto para produção de celulose é realizada com base na densidade básica e no rendimento em celulose por cozimento Kraft convencional, o que funciona bem para o ordenamento de clones, mas não é suficiente para avaliar o desempenho geral da madeira numa fábrica de celulose Kraft branqueada (FERREIRA et al., 2006).

De acordo com Alves et. al. (2011) para que o Brasil continue a aumentar a produção de celulose será indispensável à contínua melhoria de produtividade das florestas clonais de eucalipto e da sua qualidade, além do estabelecimento de florestas com novas espécies não convencionais no Brasil. Segundo Protásio et. al. (2014) "as características de produtividades de clones de eucalipto devem ser incorporadas aos estudos de qualidade da madeira" e o entendimento da composição e organização dos tipos celulares que compõem a madeira é um fator decisivo para que esta seja trabalhada e utilizada de forma racional (OLIVEIRA; HELLMEISTER, 1999).

O objetivo deste trabalho foi comparar a densidade básica e as dimensões anatômicas, nas duas primeiras toras e entre clones de árvores de Eucalyptus urophylla com 8 anos de idade, plantados comercialmente na região de Esplanada, na
Bahia. Essas informações contribuirão para a avaliação do potencial desses clones para a utilização da espécie, principalmente na indústria de papel celulose.

\section{Materiais e Métodos}

O material utilizado nesta pesquisa foi obtido de várias populações experimentais de clones de Eucalyptus urophylla S.T.Blake (Tabela 1), procedentes da região de Esplanada, no estado da Bahia. O município de Esplanada localiza-se a uma latitude de $11^{\circ} 47^{\prime} 46^{\prime \prime}$ sul, longitude $37^{\circ} 56^{\prime} 42^{\prime \prime}$ oeste, com uma altitude de 142 metros O clima na região é predominantemente tropical, com temperaturas elevadas e médias anuais que ultrapassam os $26^{\circ} \mathrm{C}$, a precipitação média é $1.500 \mathrm{~mm}$ anuais (CLIMA BAHIA, 2015).

Inicialmente foi realizado um inventário e a partir da distribuição diamétrica obtida, foi eleita a classe de DAP (diâmetro a altura do peito) mediana de cada população (Tabela 1).

Tabela 1. Idade, diâmetro a altura do peito (DAP) e altura total (HT) dos clones.

Table 1. Age, breast height diameter (DAP) and total height (HT) of the clones.

\begin{tabular}{ccccc}
\hline Tratamentos & Clone & $\begin{array}{c}\text { Idade } \\
\text { (anos) }\end{array}$ & $\begin{array}{c}\text { DAP } \\
(\mathbf{c m})\end{array}$ & $\begin{array}{c}\text { HT } \\
(\mathbf{m})\end{array}$ \\
\hline 1 & COP1249 & 8 & 31,7 & 30,2 \\
2 & COP1404 & 8 & 35,4 & 40,7 \\
3 & COP1406 & 8 & 31,5 & 36,0 \\
4 & COP1296 & 8 & 29,9 & 36,7 \\
\hline
\end{tabular}

Para a avaliação das propriedades da madeira selecionouse 16 árvores, sendo quatro de cada um dos tratamentos, que foram devidamente identificadas no campo. De cada árvore selecionada foram tomadas duas toras de 3 metros de comprimento, sendo a $1^{\mathrm{a}}$ tora, retirada na base da árvore e a $2^{\mathrm{a}}$ tora, subsequente.

As toras foram desdobradas e produziram tábuas. Dessas tábuas foram retiradas as amostras de madeira para os ensaios da densidade básica e dimensões celulares. De cada uma das 
toras foram retiradas duas amostras de $2,5 \times 10 \times 12 \mathrm{~cm}$, da $2^{\mathrm{a}}$ tábua do sentido casca-medula da $1^{\mathrm{a}}$ e $2^{\mathrm{a}}$ tora (Figura 1).

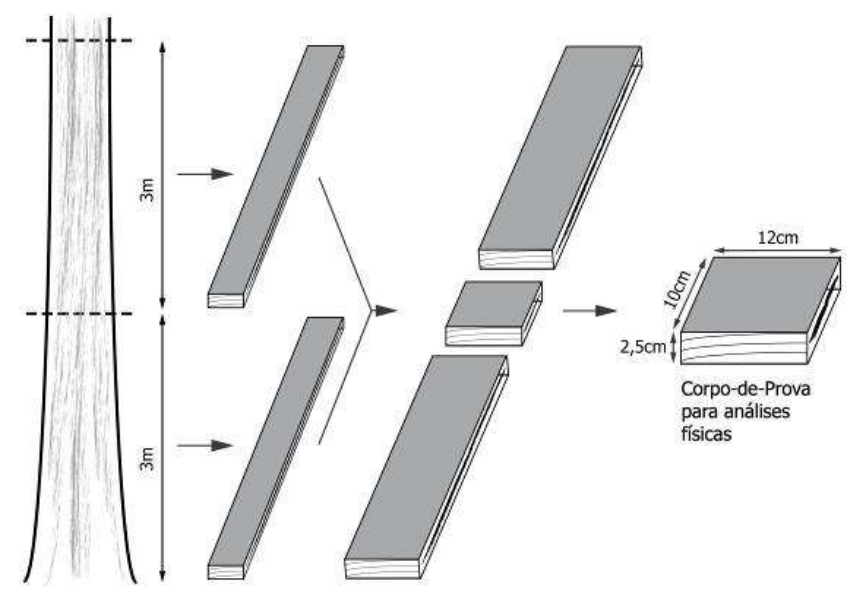

Figura 1. Representação esquemática da obtenção das amostras de madeira.

Figure 1. Schematic representation of wood sample preparation.

As amostras obtidas foram transformadas em corpos de prova para estudo da densidade básica (DB), comprimento de elemento de vaso $(\mathrm{CV})$, comprimento de fibra (CF), diâmetro de fibra (DF), lume de fibra (LF), espessura da parede da fibra (EPF), diâmetro de elemento de vaso (DV), frequência de elemento de vaso $(\mathrm{FV})$, altura de raio $(\mathrm{AR})$, diâmetro de raio (DR), frequência de raio (FR) e porcentagem total de elemento de vaso (PTV).

Para a obtenção da densidade básica (DB), foi utilizado o método da balança hidrostática de acordo com a NBR 11941(ABNT, 2003). Os corpos de prova de 2 × 2 x $3 \mathrm{~cm} \mathrm{ob-}$ tidos foram saturados em água por um período de aproximadamente um mês, o que possibilitou a obtenção da massa saturada e imersa de cada amostra. Posteriormente esses corpos de prova foram secos em estufa até atingirem a massa seca constante a $105 \pm 3^{\circ} \mathrm{C}$.

Também foram confeccionados outros corpos de prova de dimensões de $2 \times 2 \times 3 \mathrm{~cm}$, destes retiraram-se pequenos fragmentos para serem macerados segundo o método de
Franklin modificado (BERLYN ; MIKSCHE, 1976). As mensurações foram realizadas em microscópio equipado com câmera digital e computador com software de análise de imagens. Retiraram-se fotografias de 25 diferentes vasos e fibras, utilizando-se o programa Image-Pro Plus 6.0 para medição de comprimento de vasos, comprimento de fibra, diâmetro de fibra, lume e espessura de parede das fibras.

Para calcular a espessura da parede das fibras, utilizou-se a equação 1 .

$$
E P F=\frac{D F-L F}{2}
$$

Em que: EPF: espessura da parede da fibra $(\mu \mathrm{m})$; DF: diâmetro da fibra $(\mu \mathrm{m})$; LF: diâmetro do lume da fibra $(\mu \mathrm{m})$.

Para a análise da frequência e diâmetro dos vasos e também frequência e dimensões dos raios corpos de prova com cerca de $2 \mathrm{~cm}^{3}$ foram amolecidos em solução de água, álcool e glicerina por um período de duas horas e secções de 18 a 25 $\mu \mathrm{m}$ foram obtidas em micrótomo de deslize para a obtenção de lâminas temporárias. As secções foram clarificadas com hipoclorito de sódio $60 \%$ e coradas com safranina aquosa $1 \%$ (SASS, 1951).

Lâminas provisórias foram preparadas para a mensuração das características anatômicas segundo o IAWA Committee (1989). Todas as mensurações foram realizadas em microscópio equipado com câmera digital e computador com software de análise de imagens.

A porcentagem total de vaso (PTV) foi obtida de acordo com a equação 2 .

$$
P T V=\left[\frac{\sum_{i=1}^{n} A T V}{A T}\right] 100
$$

Em que: PTV: porcentagem total de área de vaso (\%); ATV: somatória total da área de vaso $\left(\mathrm{mm}^{2}\right)$; AT: área total $\left(1 \mathrm{~mm}^{2}\right)$. 
Após a obtenção dos valores das dimensões das fibras foram calculados também, o Fator de Runkel (R) e o Coeficiente de rigidez (CR) e para isso utilizou-se as equações 3 e 4 respectivamente, de acordo com Paula e Alves (2007).

$$
R=\frac{(2 e)}{d}
$$

Em que: $\mathrm{R}=$ Fator de Runkel; e = espessura da parede da fibra $(\mu \mathrm{m}) ; \mathrm{d}=$ diâmetro externo da fibra $(\mu \mathrm{m})$.

$$
C R=\left(\frac{d}{D}\right) 100
$$

Em que: $\quad \mathrm{CR}=$ Coeficiente de rigidez (\%); $\mathrm{d}=$ diâmetro interno da fibra $(\mu \mathrm{m}) ; \mathrm{D}=$ diâmetro externo da fibra $(\mu \mathrm{m})$.

A análise estatística foi desenvolvida com auxílio do programa SAS Procedures Guide (1999). Os resultados foram avaliados por meio de análise de variância (ANOVA) e teste Tukey a 5\% de probabilidade de erro para detectar as variações.

\section{Resultados e Discussão}

Na Tabela 2 há o resumo da análise de variância da densidade básica e as dimensões dos elementos celulares da madeira de eucalipto, em função do clone e da posição da tora, na altura da árvore. Ocorreram diferenças significativas entre os clones para: densidade básica, frequência de vaso, porcentagem total de vaso, altura de raio e diâmetro de raio. A posição da tora na altura da árvore diferiu significativamente somente para a frequência de vaso.

De maneira geral, ocorreram variações entre os clones para a densidade básica e para a maioria das dimensões celulares da madeira, porém para posição das toras foi parcialmente aceita porque houve diferença significativamente somente para a frequência de elemento de vaso.
Segundo os valores de densidade básica da madeira obtida, os mesmos podem ser classificados como de baixa a média densidade (Tabela 3) (ZENID, 2007). Resultados semelhantes foram obtidos por Trugillho et al. (2007) para vários clones de Eucalyptus com 6 anos e Vale et al. (2002) em clones de Eucalyptus urophylla, também com 6 anos de idade. O clone COP1296 apresentou o maior valor de densidade e o clone COP1406, o menor, o que diferenciou significativamente os dois materiais (Tabela 3). As toras 1 e 2 não se diferenciaram significativamente para a densidade básica (Tabela 3).

O comprimento de fibra também não apresentou diferença significativa entre clones e entre toras. O clone COP 1249 tem o maior comprimento de fibra com $1.090 \mu \mathrm{m}$ e o COP1406 o menor com $1.015 \mu \mathrm{m}$ (Tabela 3). Para efeito comparativo verificou-se que a literatura apresenta valores entre 500-1.700 $\mu \mathrm{m}$ em Eucalyptus benthamii com idade de sete anos (NISGOSKI et al, 1998) e $1.276 \mu \mathrm{m}$ em Eucalyptus grandis, aos 16 anos de idade (BRASIL; FERREIRA, 1972). De acordo com Bhat et al. (1990), a taxa de crescimento do comprimento das fibras se estabiliza por volta de 9 anos de idade, o que é um indicativo que a rotação do plantio para o uso da indústria de celulose, pode ser abaixo dessa idade.

A densidade básica e as dimensões das fibras apresentaram poucas variabilidades, o que segundo Gonçalez et al. (2014), do ponto de vista industrial, é interessante devido o material ter boa homogeneidade nas suas características anatômicas e a densidade básica. Essa homogeneidade favorece a impregnação e remoção da lignina dos cavacos nos processos de cozimento e, por consequência, na formação do papel haverá uma melhor ligação entre fibras, ganhando o papel em qualidade.

De acordo com a literatura, normalmente para as espécies de Eucalyptus ocorre um aumento da densidade básica e com- 
primento das fibras no sentido medula casca, com uma tendência de estabilização nas proximidades da região da casca (e.g. BHAT et al., 1990, VALE et al. 2002 e GITHIOMI;
KARIUKI, 2010). Pereira et. al; (2013) verificaram para clones de Eucalyptus urophylla, que aos 7 anos de idade, ocorre uma relação cerne/alburno da ordem de 0,76.

Tabela 2. Resumo da análise de variância efetuada para Densidade básica (DB), comprimento de fibra (CF), espessura da parede da fibra (EPF), comprimento de vaso (CV), diâmetro de vaso (DV), frequência de vaso (FV), porcentagem total de vaso (PTV), altura de raio (AR), diâmetro de raio (DR) e frequência de raio (FR) de clones de Eucalyptus urophylla aos 8 anos de idade.

Table 2. Summary of analysis of variance of basic density (DB), fiber length (CF), fiber wall thickness (EPF), vessel element length $(\mathrm{CV})$; vessel diameter (DV), vessel frequency (FV), percentage of total vessel (PTV), ray height (AR), ray width (LR) and ray frequency (FR) of 8-year old in clones of Eucalyptus urophylla.

\begin{tabular}{|c|c|c|c|c|c|c|c|c|c|c|c|}
\hline $\begin{array}{l}\text { Causa de } \\
\text { variação }\end{array}$ & GL & $\begin{array}{c}\mathrm{DB} \\
\left(\mathrm{g} \mathrm{cm}^{-3}\right)\end{array}$ & $\begin{array}{c}\text { CF } \\
(\mu \mathrm{m})\end{array}$ & $\begin{array}{l}\text { EPF } \\
(\mu \mathrm{m})\end{array}$ & $\begin{array}{c}\text { CV } \\
(\mu \mathrm{m})\end{array}$ & $\begin{array}{c}\text { DV } \\
(\mu \mathrm{m})\end{array}$ & $\begin{array}{c}\mathrm{FV} \\
\left(\mathrm{n}^{\mathrm{o}} \mathrm{mm}^{-1}\right)\end{array}$ & $\begin{array}{c}\text { PTV } \\
(\%)\end{array}$ & $\begin{array}{c}\text { AR } \\
(\mu \mathrm{m})\end{array}$ & $\begin{array}{c}\text { DR } \\
(\mu \mathrm{m})\end{array}$ & $\begin{array}{c}\text { FR } \\
\left(\mathrm{n}^{\mathrm{o}} \mathrm{mm}^{-1}\right)\end{array}$ \\
\hline Clone (C) & 3 & $0,0130^{* *}$ & $8230^{\text {n.s }}$ & $0,12^{\mathrm{n} . \mathrm{s}}$ & $16266^{\text {n.s }}$ & $73^{n . s}$ & $8,19^{*}$ & $\begin{array}{c}52809 \\
1^{*}\end{array}$ & $\begin{array}{c}8787 \\
*\end{array}$ & $48,46^{*}$ & $2,21^{\mathrm{n} . \mathrm{s}}$ \\
\hline Tora $(\mathrm{T})$ & 1 & $0,0003^{\text {n.s. }}$ & $10548^{\mathrm{n} . \mathrm{s}}$ & $0,01^{\mathrm{n} . \mathrm{s}}$ & $\begin{array}{c}33735 \\
\text { n.s }\end{array}$ & $72^{\text {n.s }}$ & $13,46^{*}$ & $531^{\mathrm{n} . s}$ & $\begin{array}{c}603 \\
\text { n.s }\end{array}$ & $\begin{array}{c}1,31 \\
\text { n.s }\end{array}$ & $0,55^{\text {n.s }}$ \\
\hline $\mathrm{C} \times \mathrm{T}$ & 3 & $0,0036^{\text {n.s. }}$ & $1829^{\text {n.s }}$ & $0,58^{\text {n.s }}$ & $\begin{array}{c}24948 \\
\text { n.s }\end{array}$ & $372^{\text {n.s }}$ & $0,65^{\mathrm{n} . \mathrm{s}}$ & $\begin{array}{c}10843 \\
2^{\text {n.s }}\end{array}$ & $\begin{array}{c}1299 \\
\text { n.s }\end{array}$ & $\begin{array}{c}5,14 \\
\text { n.s }\end{array}$ & $0,78^{n . s}$ \\
\hline Resíduo & 24 & 0,0032 & 7470 & 0,72 & 14556 & 101 & 1,14 & 44926 & 1225 & 9,40 & 0,95 \\
\hline Média & & 0,49 & 1059 & 5,34 & 618 & 134 & 9,02 & 12,49 & 308 & 17,07 & 11,55 \\
\hline $\mathrm{DP}$ & & 0,0641 & 84 & 0,79 & 127 & 11 & 1,47 & 3,10 & 44 & 3,53 & 1,02 \\
\hline $\mathrm{CV}_{\mathrm{e}}(\%)$ & & 11,51 & 8,15 & 15,98 & 19,51 & 7,49 & 11,85 & 16,95 & $\begin{array}{c}11,3 \\
4\end{array}$ & 17,95 & 8,44 \\
\hline
\end{tabular}

Onde: ${ }^{* *}$ significativo ao nível de $1 \%$ de significância; ${ }^{*}$ significativo ao nível de $5 \%$ de significância; $n . s$ = não significativo; DP = desvio padrão; $\mathrm{CVe}=$ coeficiente de variação experimental; $\mathrm{GL}=$ Grau de liberdade.

Tabela 3. Média para densidade básica (DB), comprimento de fibra (CF), espessura da parede da fibra (EPF), comprimento de vaso $(\mathrm{CV})$, diâmetro de vaso $(\mathrm{DV})$, frequência de vaso $(\mathrm{FV})$, porcentagem total de vaso $(\mathrm{PTV})$, altura de raio (AR), diâmetro de raio (DR) e frequência de raio (FR) em clones de Eucalyptus urophylla aos 8 anos de idade.

Table 3. Average of basic density (DB), fiber length (CF), fiber wall thickness (EPF), vessel element length (CV); vessel diameter (DV), vessel frequency (FV), percentage of total vessel (PTV), ray height (AR), ray width (LR) and ray frequency (FR) of 8year old in clones of Eucalyptus urophylla.

\begin{tabular}{|c|c|c|c|c|c|c|c|c|c|c|}
\hline Tratamento & $\begin{array}{c}\mathrm{DB} \\
\left(\mathrm{g} \mathrm{cm}^{-3}\right)\end{array}$ & $\begin{array}{c}\text { CF } \\
(\mu \mathrm{m})\end{array}$ & $\begin{array}{l}\mathrm{EPF} \\
(\mu \mathrm{m})\end{array}$ & $\begin{array}{c}\text { CV } \\
(\mu \mathrm{m})\end{array}$ & $\begin{array}{c}\text { DV } \\
(\mu \mathrm{m})\end{array}$ & $\begin{array}{c}\mathrm{FV} \\
\left(\mathrm{n} \mathrm{mm}^{-2}\right)\end{array}$ & $\begin{array}{c}\text { PTV } \\
(\%)\end{array}$ & $\begin{array}{c}\text { AR } \\
(\mu \mathrm{m})\end{array}$ & $\begin{array}{c}\text { DR } \\
(\mu \mathrm{m})\end{array}$ & 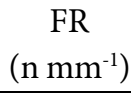 \\
\hline COP1249 & $0,52^{\mathrm{ab}}$ & $1090^{\mathrm{a}}$ & $5,3^{\mathrm{a}}$ & $597^{a}$ & $134^{\mathrm{a}}$ & $9,6^{a}$ & $16,10^{\mathrm{a}}$ & $327^{a}$ & $17,7^{\mathrm{ab}}$ & $11,2^{\mathrm{a}}$ \\
\hline COP1404 & $0,47^{\mathrm{ab}}$ & $1072^{\mathrm{a}}$ & $5,2^{\mathrm{a}}$ & $655^{\mathrm{a}}$ & $135^{\mathrm{a}}$ & $7,2^{\mathrm{b}}$ & $12,01^{\mathrm{b}}$ & $334^{\mathrm{a}}$ & $13,6^{\mathrm{b}}$ & $11,1^{\mathrm{a}}$ \\
\hline COP1406 & $0,45^{\mathrm{b}}$ & $1015^{\mathrm{a}}$ & $5,2^{\mathrm{a}}$ & $655^{\mathrm{a}}$ & $137^{\mathrm{a}}$ & $9,6^{\mathrm{a}}$ & $11,88^{\mathrm{b}}$ & $261^{\mathrm{b}}$ & $19,5^{\mathrm{a}}$ & $11,4^{\mathrm{a}}$ \\
\hline COP1296 & $0,54^{\mathrm{a}}$ & $1061^{\mathrm{a}}$ & $5,5^{\mathrm{a}}$ & $564^{\mathrm{a}}$ & $130^{\mathrm{a}}$ & $9,2^{\mathrm{a}}$ & $9,99^{\mathrm{b}}$ & $311^{\mathrm{a}}$ & $17,3^{\mathrm{ab}}$ & $12,3^{\mathrm{a}}$ \\
\hline $1^{\text {a }}$ tora & $0,50^{\mathrm{a}}$ & $1078^{\mathrm{a}}$ & $5,3^{\mathrm{a}}$ & $585^{\mathrm{a}}$ & $135^{\mathrm{a}}$ & $8,3^{\mathrm{b}}$ & $12,45^{\mathrm{a}}$ & $312^{\mathrm{a}}$ & $17,2^{\mathrm{a}}$ & $11,6^{\mathrm{a}}$ \\
\hline $2^{\text {a }}$ tora & $0,49^{a}$ & $1041^{\mathrm{a}}$ & $5.3^{\mathrm{a}}$ & $650^{\mathrm{a}}$ & $132^{\mathrm{a}}$ & $9,6^{\mathrm{a}}$ & $12,53^{\mathrm{a}}$ & $304^{\mathrm{a}}$ & $16,8^{\mathrm{a}}$ & $11,4^{\mathrm{a}}$ \\
\hline
\end{tabular}

Médias seguidas de letras diferentes em uma mesma coluna diferem entre si (ao nível de $5 \%$ de probabilidade de erro).

O clone COP1296 apresentou maior espessura de parede das fibras (EPF) com 5,52 $\mu \mathrm{m}$, porém não se observou diferenças significativas entre toras e entre clones a 5\% de significância, esse resultado é semelhante ao obtidos por Gonçalez et al. (2014), para uma população de E. urograndis também com 8 anos de idade e Evangelista et. al. (2010) para clone de E. urophylla também com 8 anos de idade. Fibras com paredes mais espessas como o clone COP1296 possuem maior teor relativo de celulose do que fibras de paredes mais finas, existindo uma correlação positiva entre a espessura da parede 
e o rendimento em produção de pasta de celulose (PAULA, 1999).

A Tabela 3 mostra que o clone COP1296 apresentou menor comprimento de vaso com $564 \mu \mathrm{m}$, é o clone COP1404 o maior comprimento de vaso com $655 \mu \mathrm{m}$, assim como a tora 2 com comprimento de vaso de $650 \mu \mathrm{m}$, porém não apresentaram diferenças significativas entre clones e as posições das toras na altura da árvore. Nisgoski et al. (1998), verificaram que o comprimento vascular de populações de Eucalyptus benthamii aos sete anos de idade variaram de 120 a $650 \mu \mathrm{m}$, sendo menores do que os obtidos nessa pesquisa. Os vasos grandes verificados nestes clones favorecem algumas características desses eucaliptos e desfavorecem outras, como nos processos de conversão para celulose, são excelentes para favorecer a impregnação dos cavacos. Porém, suas celuloses apresentam mais acentuadamente um defeito conhecido como arrancamento ou arrepelamento de vasos, quando utilizadas na fabricação de papéis de impressão.

A variável diâmetro de elemento de vaso (DV) não apresentou diferenças significativas entre clone e entre toras a $5 \%$ de significância, tendo maior valor de $137,5 \mu \mathrm{m}$ no clone COP1406 e menor de 130,4 $\mu$ m no COP1296. Trabalho com Eucalytpus grandis com 6,9 anos de idade mostra valores de $88,4 \mu \mathrm{m}$ a $120,7 \mu \mathrm{m}$ para (DV) (BELINI et al., 2008) e Evangelista et. al. (2010) para clone de Eucalyptus urophylla com 8 anos de idade, obtiveram valor de $115 \mu \mathrm{m}$ para o diâmetro de elemento de vaso.

Na frequência de vaso (FV), o clone COP1404 diferiu do restante dos clones, apresentando FV igual a $7,5 \mathrm{n} \mathrm{mm}^{-2}$, a tora 2, também diferiu da tora 1 , com FV de $9,7 \mathrm{n} \mathrm{mm}^{-2}$. Belini et al. (2008) em um estudo com Eucalyptus grandis obtiveram valores de FV variando entre $8,9 \mathrm{n} \mathrm{mm}^{-2}$ a $16,2 \mathrm{n} \mathrm{mm}^{-2}$.

O clone COP1406 apresentou diferença a 5\% de significância na altura de raio (AR), com o menor valor de $261 \mu \mathrm{m}$ e o clone COP1404 com $334 \mu \mathrm{m}$, sendo o de maior dimensão.
As toras 1 e 2 não diferiram entre si para AR. Rocha et al. (2004) em um trabalho com Eucalyptus grandis obtiveram valores de AR entre $203 \mu \mathrm{m}$ na região da medula e $201 \mu \mathrm{m}$ na proximidade da casca.

Os clones COP1404 e COP1406 diferiram entre si quanto ao diâmetro de raio (DR). O COP1406 apresentou 19,55 $\mu \mathrm{m}$ e COP1404 13,67 $\mu \mathrm{m}$. As toras 1 e 2 não apresentaram diferenças significativas, Rocha et al. (2004) estudando Eucalyptus grandis verificaram valores de DR iguais a $12,97 \mu \mathrm{m}$ na medula e $12,6 \mu \mathrm{m}$ na casca.

Para frequência de raio (FR) observou-se que não houve diferenças entre clones e entre toras, tendo valores entre 11,2

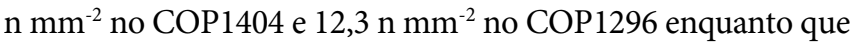
Rocha et. al. (2004) verificaram que a frequência de raio em $E$ ucalyptus grandis variam de $11,13 \mathrm{n} \mathrm{mm}^{-2}$ na medula e $9,13 \mathrm{n}$ $\mathrm{mm}^{-2}$ na casca. O clone COP1249 apresentou o maior valor de porcentagem de vaso total (PTV) igual a 16,09\%.

De acordo com resultados apresentados verifica-se que o clone COP1249 apresentou os maiores valores de comprimento de fibra, porém não diferiu significativamente dos outros clones. Essa característica que deve ser levada em consideração, pois a diferença de comprimento é determinante na resistência dos papéis à tração, ao arrebentamento e ao rasgo (BRITO et al., 1976). As mudanças no comprimento das fibras variam entre espécies, porém, o mais comum é observar fibras mais longas na base das árvores, esta proporção pode ser explicada pelo aumento na proporção de lenho juvenil no sentido base para o topo (ZOBEL; BUIJTENEN, 1989).

As dimensões das fibras interferem diretamente na densidade da madeira influenciando a massa da polpa na produção de papel (DOWNES et al., 1997). Assim, fibras maiores e com paredes mais espessas podem indicar madeiras com maior densidade, embora esta propriedade também possa ser influenciada pela frequência, diâmetro dos vasos e volume dos raios (FUJIWARA et al., 1991; FUJIWARA, 1992). 
A espessura da parede e lume das fibras são os fatores que mais influenciam na densidade da madeira (PANSHIN; DE ZEEUW, 1980). Os valores encontrados para espessura da parede da fibra estão em conformidade com os normalmente encontrados na literatura, que variam de 2,5 a 6,0 $\mu \mathrm{m}$. Polpas com características de menores espessuras de paredes celulares e maior diâmetro de lume das fibras, como o COP1406, apresentam maior potencial de colapso e menor potencial para drenagem em máquina de celulose e papel (FERREIRA et al., 2006).

De acordo com os resultados observados nota-se que o clone que teve menor frequência de vaso foi o COP1404. Esses valores também estão em conformidade com os normalmente encontrados na literatura. Estatisticamente não houve diferenças significativas entre clones e toras. Maiores diâmetros dos vasos normalmente estão associados a menores frequências (BAMBER et al., 1982; VITAL; DELLA LUCIA, 1987). Os vasos são muito importantes tanto para a fisiologia e crescimento das árvores, como para os processos de conversão da madeira em celulose. Sua presença nas madeiras de folhosas favorece a penetração e impregnação dos cavacos de folhosas pelo licor de cozimento kraft. Entretanto, não é desejável que a madeira utilizada para produção de celulose apresente alto teor dessas células, pois alto teor de vasos implica em madeira de menor densidade proporcionando menor produtividade na fábrica e maior consumo específico de madeira (ALVES et al. 2011).

Após a fabricação de celulose, a presença dos vasos pode ou não significar problemas no uso dessa celulose, dependendo do tipo de papel a ser produzido. Há tipos de papéis em que a presença dos vasos quase não causa problemas, isso acontece com papéis fabricados com polpas bem refinadas, quando os vasos acabam colapsados e fragmentados. As melhores dimensões para vasos são aqueles em que a relação entre seu comprimento e seu diâmetro seja igual ou maior que
8 a 10, ou seja, vasos longos em relação ao diâmetro parecem e se comportam mais como fibras na fabricação de papel (FOELKEL, 2009).

A relação entre comprimento e diâmetro de cada célula de vaso determina importantes características desses elementos para o uso das madeiras para fabricação de celulose para papel. Vasos que se assemelham às fibras (maior relação comprimento/diâmetro) são mais indicados, pois aderem e são retidos mais fortemente na rede que é a folha de papel (FOELKEL, 2009). Os valores dimensionais dos vasos são importantes no lenho funcional, ou seja, na condução de líquidos, e nas propriedades de utilização da madeira, afetando a qualidade superficial dos papéis de impressão (SETTE JUNIOR et al., 2009). Em geral existem cerca de 3 a 25 vasos por $\mathrm{mm}^{2}$ para espécies do gênero Eucalyptus. Há também muita variação entre as dimensões desses elementos de vaso, mas na sua maioria, esses elementos possuem diâmetro entre 60 a $250 \mu \mathrm{m}$ e comprimento entre 200 a $600 \mu \mathrm{m}$. A área de vaso total pode variar entre $22090 \mu \mathrm{m}^{2}$ a $360795 \mu \mathrm{m}^{2}$, dependendo do número de vasos por $\mathrm{mm}^{2}$ (FOELKEL, 2009). Existe uma importante variação nas porcentagens dos volumes de vasos nas madeiras de algumas espécies de eucalipto. Isso é mais em função do diâmetro médio dos vasos do que de sua frequência.

Na maioria das espécies comerciais e clones de eucalipto, a proporção de vaso no volume da madeira está entre 10 a $20 \%$. As dimensões e a frequência dos vasos variaram com os clones e com as diferenças ambientais em que crescem as árvores. Há espécies de eucaliptos com rápido crescimento e com baixa densidade básica e tendem a ter vasos bastante largos, mas às vezes não tão numerosos, como é o caso do $E$ ucalyptus deglupta e Eucalyptus grandis (FOELKEL, 2009).

Para os raios não é possível estabelecer um padrão entre as diferentes espécies de eucalipto (FLORSHEIM et al., 2009). $\mathrm{Na}$ base encontram-se os maiores valores das dimensões dos raios (FLORSHEIM et al., 2000). Há presença de raios mais 
altos e menos frequentes a 50\% da altura, a largura dos raios é maior na base (ROCHA et al., 2004). De acordo com FLORSHEIM et al., (2000) há uma tendência dos raios ficarem mais altos e largos no sentido da medula para a casca, com menores frequências.

A Tabela 4 mostra os resultados obtidos para Fator Runkel e Coeficiente de Rigidez.

Tabela 4. Índices indicativos da qualidade da polpa celulósica.

Table 4. Indicative indices of cellulose pulp quality.

\begin{tabular}{ccc}
\hline $\begin{array}{c}\text { Causas de } \\
\text { variação }\end{array}$ & $\begin{array}{c}\text { Fator de } \\
\text { Runkel }\end{array}$ & $\begin{array}{c}\text { Coeficiente de } \\
\text { Rigidez }(\%)\end{array}$ \\
\hline COP1249 & 1,04 & 51,0 \\
COP1404 & 0,89 & 53,2 \\
COP1406 & 0,88 & 53,6 \\
COP1296 & 1,21 & 45,5 \\
\hline Tora 1 & 0,92 & 52,3 \\
Tora 2 & 1,07 & 49,5 \\
\hline
\end{tabular}

Quanto maior o valor de Fator de Runkel, menos apta a madeira para a fabricação de papel. Consideram-se ideais os valores menores do que 1 , o que pode ser observado para os clones COP1404 e COP1406 e para a tora 1. Quanto maior o Coeficiente de Rigidez, maior será o achatamento e melhor o amoldamento das células. Um alto valor significa a existência de células de paredes mais finas (BURGER RICHTER, 1991). O maior valor para o Coeficiente de Rigidez foi com o clone COP1406 com 53,64\%.

\section{Conclusões}

Foi possível concluir que:

- Entre os clones ocorreram diferenças para: densidade básica, frequência de vaso, porcentagem total de vaso, altura de raio e diâmetro de raio e. A posição da tora na altura da árvore diferiu significativamente somente para a frequência de vaso.
- O clone COP1406 apresentou os maiores valores para: diâmetro de raio e fibras com menor espessura da parede.

- Para comprimento de vaso e diâmetro de fibra os clones COP1404 e COP1406 apresentaram os maiores valores.

- O comprimento de fibra foi maior para o COP1249.

- As maiores medidas de espessura da parede de fibra e frequência de raio foram com o clone COP1296;

- A maior altura de raio e porcentagem total de raio foi para o clone COP1404.

- A tora 1 apresentou maiores valores no comprimento de fibra, diâmetro de vaso, altura de raio diâmetro de raio e frequência de raio.

\section{Referências}

ALVES, I. C. N.; GOMIDE, J. L.; COLODETTE, J. L.; SILVA, H. D. Caracterização tecnológica da madeira de Eucalyptus benthamii para produção de celulose Kraft. Ciência Florestal, Santa Maria, v. 21, n. 1, p. 167-74, 2011.

ASSOCIAÇÃO BRASILEIRA DE NORMAS TÉCNICAS. NBR 11941: Madeira: determinação da densidade básica. Rio de Janeiro, 2003.

BAMBER, R. K.; HORNE, R.; GRAHAN-HIGGS, A. Effect of fast growth on the wood properties of Eucalyptus grandis. Australian Forest Research, Melbourne, n. 12, p. 163-167, 1982.

BELINI, U. L.; TOMAZELLO FILHO, M.; CHAGAS, M. P.; DIAS, C. T. DOS S. Caracterização da estrutura anatômica, densidade básica e morfologia de cavacos da madeira de Eucalyptus grandis para a produção de painéis MDF. Revista Árvore, Viçosa, v. 32, n. 4. p. 707-713, 2008.

BERLYN, G. P.; MIKSCHE, J. P. Botanical microtechnique and cytochemistry. The Iowa University Press, Iowa, 1976.

BHAT, K. M.; BHAT, K. V.; DHAMODARAN, T. K. Wood density and fibre length of Eucalyptus grandis grown in Kerala, India. Wood and Fiber Science, Madison, v. 22, n. 1, p. 54-61, 1990.

BRASIL, M. A. M.; FERREIRA, M. Variações da densidade básica e das características das fibras em Eucalyptus grandis 
Hill ex Maiden ao nível do DAP. IPEF, Piracicaba, n. 5, p. 8190, 1972.

BRITO, J. O; BARRICHELO, L. E. G.; FERREIRA, M. O melhoramento dos caracteres da madeira frente a produção de celulose e papel. IPEF Boletim Informativo, Piracicaba, v. 6 , n. 19 , p. 96-115, 1976.

BURGER, L. M.; RICHTER, H. G. Anatomia da madeira. São Paulo: Nobel, 1991.

CLIMA BAHIA. Disponível em: < http://www.apolo11.com/>. Acessado em: 14 outubro 2015.

DOWNES, G. M.; HUDSON. I. L.; RAYMOND, C. A.; DEAN, G. H.; MICHELL, A. J.; SCHIMLECK, L. R.; EVANS, R.; MUNERI, A. Sampling plantation eucalypts for wood and fibre properties. Australia: CSIRO Publishing, 1997.

EVANGELISTA, W. V.; CASTRO SILVA, J.; VALLE, M. L. A.; XAVIER, B. A. Caracterização anatômica quantitativa da madeira de clones de Eucalyptus camaldulensis Dehnh. e Eucalyptus urophylla S.T. Blake. Scientia Forestalis, Piracicaba, v. 38, n. 86, p. $273-284,2010$.

FERREIRA, C. R.; JUNIOR, M. F.; COLODETE, J. L.; GOMIDE, J. L.; CARVALHO, A. M. M. L. Avaliação tecnológica de clones de eucalipto: parte 1 - qualidade da madeira para produção de celulose Kraft. Scientia Forestalis, Piracicaba, v. 70, n. 1, p .161-170, 2006.

FLORSHEIM, S. M. B.; COUTO, H. T. Z.; LIMA, I. L.; LONGUI; E. L. Variação nas dimensões dos elementos anatômicos da madeira de Eucalyptus dunnii aos sete anos de idade. Revista Instituto Florestal, São Paulo, v. 21, n. 1, p. 79-91, 2009.

FLORSHEIM, S. M. B.; COUTO,H.T.Z.; SPEGIORIN,L.; ROCHA,F.T.. Variação da estrutura anatômica da madeira de Eucalyptus saligna aos sete anos. Revista Instituto Florestal, São Paulo, v. 12, n. 2, p. 170-191, 2000.

FOELKEL, C. Elementos de vaso e celuloses de Eucalipto. Associação Brasileira Técnica de Celulose e Papel, Grau Celsius, 2009. Disponível em: http://www.eucalyptus.com.br/capitulos/PT04_vasos.pdf Disponível em: Acesso em: 12 dez. 2016.

FUKIWARA, S.; SAMESHIMA, K.; KURODA, K.; TAKAMURA, N. Anatomy and properties of Japanese hardwoods I. Variation of fibre dimensions and tissue proportions and their relation to basic density. IAWA Bulletin, Leiden, n.12, p. 419-424, 1991.
FUJIWARA S. Anatomy and properties of Japanese hardwoods II. Variation of dimensions of ray cells and their relation to basic density. IAWA Bulletin, Leiden, n. 13, p.397402, 1992.

GITHIOMI, J. K.; KARIUKI, J. G. Wood basic density of Eucalyptus grandis from plantations in Central Rift Valley, Kenya: variation with age, height level and between sapwood and heartwood. Journal of Tropical Forest Science, Kuala Lumpur, v. 22, n. 3, p. 281286, 2010.

GONÇALVEZ, J. C.; SANTOS, G. L.; SILVA JUNIOR, F. G.; MARTINS, I. S.; COSTA, J. A. Relações entre dimensões de fibras e de densidade da madeira ao longo do tronco de Eucalyptus urograndis. Scientia Forestalis, Piracicaba, v. 42, n. 101, p. 81-9, 2014.

IAWA COMMITTE. IAWA list of microscopic features for hardwood identification. IAWA Bull, Leiden, n. 10, p. 219$332,1989$.

LIMA.W.P. Impacto ambiental do eucalipto. 2.ed., São Paulo: EDUSP, 1993.

LIMA, I. L. D., GARCIA, J. N., STAPE, J. L., PIEDADE, S. M. D. S. Efeito do desbaste e da fertilização nas tensões de crescimento em Eucalyptus grandis. Scientia Forestalis, Piracicaba, v. 70, p. 171-183, 2006.

MORA, A. L.; GARCIA, C. H. A Cultura de Eucalipto no Brasil. São Paulo - SP,2000.

NISGOSKI, S.; MUÑIZ, G. I. B.; KLOCK, U. Caracterização anatômica da madeira de Eucalyptus benthamii Maiden et Cambage. Ciência Florestal, Santa Maria, v. 8, n. 1, p. 67-76, 1998.

OLIVEIRA, J. T. S. HELLMEISTER, J. C. SIMÕES, J.W.; TOMAZELLO FILHO, M. Caracterização da madeira de Eucalipto para a construção civil. Scientia Forestalis, Piracicaba, n. 56, p. $113-24,1999$.

PANSHIN, A. J.; DE ZEEUW, C. Textbook of wood technology. 4.ed. New York: Mcgraw-Hill Book, 1980.

PAULA, J. E. Caracterização anatômica de madeiras nativas do cerrado com vistas à produção de madeira. Revista Cerne, Lavras, v. 5, n. 2, p. 26-40, 1999.

PAULA, J.E.; ALVES, J.L. H. Madeiras Nativas. Brasília: MOA, 2007. 
PEREIRA, B. L. C. OLIVEIRA, A. C.; CARVALHO, A.M.M.L.; CARNEIRO, A. C. O. ; VITAL, B. R. Correlações entre a relação Cerne/Alburno da madeira de eucalipto, rendimento e propriedades do carvão vegetal. Scientia Forestalis, Piracicaba, v. 41, n. 98, p. 217-25, 2013.

VITAL, B. R.; DELLA LUCIA, R. M. Effect of tree spacing on wood production and wood quality of Eucalyptus grandis and Eucalyptus urophylla at 52 months. Revista árvore, Viçosa, v. 11, p. 132-145, 1987.

PROTÁSIO,T.P.; GOULART,S.L.; NEVES,T.A.; ASSIS,M.R.; TRUGILHO,P.F. Clones comerciais de Eucalyptus de diferentes idades para o uso bioenergético da madeira. Scientia Forestalis, Piracicaba, v. 42, n. 101, p. 113-27, 2014.

ROCHA, F. T.; FLORESHEIM, S. M. B.; COUTO, H. T. Z. Variações das dimensões dos elementos anatômicos da madeira de árvores de Eucalyptus grandis Hill ex Maiden aos sete anos. Revista Instituto Florestal, São Paulo, v. 16, n.1, p. 4355, 2004.

SASS, J.E. Botanical Microtechniche. The Iowa State College Press, Ames, 1951.

S.A.S. Institute Inc. SAS Prodedures Guide.Version 8 (TSMO).SAS Institute Inc. Cary, N.C., 27513, USA, 1999.

SETTE JUNIOR, C. R.; FILHO, M. T.; LOUSADA, J. L.; LACLAU, J. P. Efeito da aplicação de fertilização nitrogenada e lodo de esgoto nas características da madeira juvenil de árvores de Eucalyptus grandis. Cerne, Lavras, v. 15, n. 3, p. 303312, 2009.

TRUGILHO, P. F.; BIANCHI, M.L.; ROSADO, S.C.S.; LIMA, J.T. Qualidade da madeira de clones de espécies e híbridos naturais de Eucalyptus. Scientia Forestalis, Piracicaba, n. 73, p. 55-62, 2007.

TRUGILHO, P. F., LIMA, J., DE PÁDUA, F., DE CARVALHO, L., ANDRADE, C. Deformação residual longitudinal (DRL) e tangencial (DRT) em seis clones de Eucalyptus spp. Cerne, Lavras, v.12, n. 3, p. 279-286, 2006.

VALE, R. S.; GRISI, R. L. M.;VENTURIN, N.; MORI, F. A. MORAIS , A. R. Efeito da desrama artificial na qualidade da madeira de clones de eucalipto em sistema agrossilvipastoril. Rev. Árvore, Viçosa-MG, v. 26, n. 3, p. 285-297, 2002.
ZENID, G. J. Madeiras e suas características. In: OLIVEIRA, José Tarcísio da Silva (Ed.); FIEDLER, N.C(Ed.); NOGUEIRA, M.(Ed.). Tecnologias aplicadas ao setor madeireiro. Vitória, ES: Jerônimo Monteiro, 2007.

ZOBEL, B. J.; BUIJTENEN, J. P. Wood variation: its causes and control. Berlin: Springer-Verlag, 1989. 363p. 